\title{
Kansas City Cardiomyopathy Questionnaire Utility in Prediction of 30-Day Readmission Rate in Patients with Chronic Heart Failure
}

\author{
Shengchuan Dai, ${ }^{1,2}$ Manoucher Manoucheri, ${ }^{1}$ Junhong Gui, ${ }^{1}$ \\ Xiang Zhu, ${ }^{3}$ Divyanshu Malhotra, ${ }^{1}$ Shenjing Li, ${ }^{4}$ Jason D'souza, ${ }^{1}$ Fnu Virkram, ${ }^{1}$ \\ Aditya Chada, ${ }^{1}$ and Haibing Jiang ${ }^{1}$ \\ ${ }^{1}$ Internal Medicine Residency Program, Department of Medicine, Florida Hospital Orlando, Orlando, FL, USA \\ ${ }^{2}$ Division of Cardiology, University of Illinois at Chicago, Chicago, IL, USA \\ ${ }^{3}$ Center for Interventional Endoscopy, Florida Hospital Orlando, Orlando, FL, USA \\ ${ }^{4}$ Division of Cardiology, University of South Dakota, Vermillion, SD, USA
}

Correspondence should be addressed to Shengchuan Dai; sdai240@gmail.com

and Manoucher Manoucheri; manoucher.manoucheri.md@flhosp.org

Received 23 May 2016; Revised 18 July 2016; Accepted 23 August 2016

Academic Editor: Stephan von Haehling

Copyright (C) 2016 Shengchuan Dai et al. This is an open access article distributed under the Creative Commons Attribution License, which permits unrestricted use, distribution, and reproduction in any medium, provided the original work is properly cited.

\begin{abstract}
Background. Heart failure (HF) is one of the most common diagnoses associated with hospital readmission. We designed this prospective study to evaluate whether Kansas City Cardiomyopathy Questionnaire (KCCQ) score is associated with 30-day readmission in patients hospitalized with decompensated HF. Methods and Results. We enrolled 240 patients who met the study criteria. Forty-eight (20\%) patients were readmitted for decompensated HF within thirty days of hospital discharge, and 192 (80\%) patients were not readmitted. Compared to readmitted patients, nonreadmitted patients had a higher average KCCQ score (40.8 versus $32.6, P=0.019$ ) before discharge. Multivariate analyses showed that a high KCCQ score was associated with low HF readmission rate (adjusted $\mathrm{OR}=0.566, P=0.022$ ). The $c$-statistic for the base model (age + gender) was 0.617 . The combination of home medication and lab tests on the base model resulted in an integrated discrimination improvement (IDI) increase of 3.9\%. On that basis, the KCQQ further increased IDI of 2.7\%. Conclusions. The KCCQ score determined before hospital discharge was significantly associated with 30-day readmission rate in patients with HF, which may provide a clinically useful measure and could significantly improve readmission prediction reliability when combined with other clinical components.
\end{abstract}

\section{Introduction}

It is estimated that heart failure (HF) affects over 5.7 million Americans with 870,000 new cases diagnosed each year. The predicted prevalence is estimated to increase $46 \%$ from 2012 to 2030 , resulting in over 8 million individuals suffering with HF [1]. The cost of caring for HF patients was about $\$ 30.7$ billion in 2012 and is estimated to increase by $127 \%$ to $\$ 69.7$ billion by 2030 [1]. Despite advances in understanding and treatment, the mortality rate of HF remains extremely high with $50 \%$ of patients dying within 5 years of initial diagnosis [2].

Readmission of HF after hospitalization is common, and unfortunately many of these readmissions are predictable and possibly preventable $[2,3]$. Although new data showed reduction in Medicare hospital readmission rates [4], HF is still one of the most common diagnoses associated with 30-day readmission; an analysis of 2007 to 2009 Medicare claims-based data showed that 24.8 percent of beneficiaries admitted with HF were readmitted within 30 days and 35.2 percent of those readmissions were for HF [5]. These concerning statistics paved the way for a stronger focus on tools to predict and prevent such readmissions.

The Kansas City Cardiomyopathy Questionnaire (KCCQ) was a tool initially designed to provide a better description of health-related quality of life in patients with HF [6]. This questionnaire identified the following clinically relevant domains: physical limitations (question 1), 
symptoms (frequency [questions 3, 5, 7, and 9], severity [questions 4, 6, and 8], and change over time [question $2]$ ), self-efficacy and knowledge (questions 11, 12), social interference (question 16), and health-related quality of life (questions 13-15) [6]. Previous studies have shown that KCCQ score correlated with survival and hospitalization in patients with HF [7] and was an independent predictor of poor prognosis in this patient population [8]. In addition, KCCQ score measured 1 week after hospital discharge independently predicted one-year survival free of cardiovascular readmission [9]. More recently, KCCQ has also been studied during acute HF hospitalization and demonstrated sensitivity to acute changes, but score changes during hospitalization did not predict short-term readmission [10], although it was a relatively small study, with a sample size of only 52 patients, and it did not investigate the relationship between KCCQ score and HF readmission. Therefore, whether KCCQ score can be used to predict the short-term readmission has yet to be completely evaluated.

To address these gaps in knowledge and explore the feasibility of using the KCCQ score to predict the short-term $\mathrm{HF}$ readmission, we designed and conducted this prospective study.

\section{Methods}

The study was approved by the Florida Hospital Institutional Review Board and conducted in accordance with the Declaration of Helsinki. The study was conducted at Florida Hospital, Orlando Campus. Patients who were admitted to the HF unit were screened and enrolled for the study. The inclusion criteria were patients admitted with decompensated $\mathrm{HF}$ with ejection fraction (EF) less than or equal to $40 \%$ and age between 20 and 89 years. Exclusion criteria were noncardiac disease with a life expectancy of less than one year, HF due to uncorrected valvular heart disease, psychiatric illness interfering with an appropriate follow-up, inability to understand study procedure, and inability to provide informed consent. Primary endpoint was 30-day readmission rate and the KCCQ score. Admission comorbid conditions, demographics, laboratory, echocardiographic data, and medications on discharge were secondary endpoints.

For every patient who met the study criteria, a trained research assistant explained the study to the patient and administered the KCCQ after a written informed consent was obtained. The assessment was generally completed within 1-3 days before discharge. A follow-up conversation was performed over the telephone 30 days after discharge to determine if rehospitalization occurred or not. Postdischarge readmission information was gathered through follow-up interview with the patient.

To evaluate associations between KCCQ score and readmission within 30 days after discharge, we first compared the difference between the nonreadmission group and readmission group in terms of the KCCQ scores, demographic characteristics, comorbidity, medications, and laboratory data using univariate analysis. In the univariate analysis, $t$-test was used for continuous variable, and Fisher's exact test was used for count number analysis. We then performed multivariate analysis to investigate how each clinical factor was associated with HF readmissions after controlling for the other factors. In the multivariate analysis, logistic regression models were used, and adjusted odds ratios (OR) were estimated for each factor hypothesized to predict HF readmission. We included $\mathrm{HF}$ readmission as a dependent variable and all potential factors as independent predictors in the logistic regression irrespective of whether they showed a significant difference between readmission and nonreadmission groups in the univariate analysis.

After the multivariate analysis, we further constructed five simplified prediction models and evaluated the importance of KCCQ score in the final model through comparing area under receiver operating characteristic curve (ROC) of each model. In this analysis, we also used integrated discrimination improvement (IDI), described by Pencina et al., to measure the average increase in model sensitivity penalized for average decrease in specificity with the addition of new variables [11]. In the prediction models, age was transformed to every 10 -year increment, ejection fraction to every $10 \%$ decrease, KCCQ score to every 25-point increment, and sodium level to binary variable $(<135$ or $\geq 135)$.

Two hundred and twenty-eight $(228$, or $95 \%)$ patients had complete data for all variables. However, 12 (5\%) patients had missing data in either age or race. As no nested missing pattern was detected, multiple imputation models were used for data imputation. As age was a continuous variable and race was a binary variable, normal linear regression was used for age while logistic regression was used for race imputation. All analyses were performed by Stata version 14 (StataCorp., 2015). All $P$ values were two-tailed, and $\alpha<0.05$ was set as the level of statistical significance for all tests.

\section{Results}

In total, 240 patients were enrolled in the study. Forty-eight (20\%) patients were readmitted within 30 days after discharge for HF while 192 (80\%) patients were not readmitted or readmitted for reasons other than HF (Table 1). There was no significant difference between the nonreadmitted and readmitted patients in terms of average age (63.0 versus $59.9 \mathrm{yrs}$, $P=0.163$ ), initial length of hospital stay (11.2 versus 9.7 days, $P=0.420)$, or percentage of white patients $(59.9 \%$ versus $56.3 \%, P=0.743)$. However, a significant difference between these two groups was noted on comparing gender, with male patients being more prone to being readmitted than female (85.4\% versus $68.8 \%$ for male and $14.6 \%$ versus $31.3 \%$ for female, $P=0.020)$. None of the comorbidities showed significant difference in the relative frequency between the readmission and nonreadmission group (Table 1).

The KCCQ score, lab test results on admission, and discharge medications were compared between the nonreadmitted and readmitted patients (Table 2). The average KCCQ score was significantly higher in the nonreadmitted patients than in readmitted patients (40.8 versus $32.6, P=0.019$ ). Compared to readmitted patients, nonreadmitted patients had a higher ejection fraction on admission $(24.7 \%$ versus $21.8 \%, P=0.021)$. However, no significant difference was detected on comparing discharge medications, blood sodium 
TABLE 1: Summary of demographic characteristics and medical history between HF readmission and nonreadmission within 30 days after discharge.

\begin{tabular}{|c|c|c|c|}
\hline \multirow{2}{*}{$\begin{array}{l}\text { Demographic } \\
\text { characteristics }\end{array}$} & \multicolumn{3}{|c|}{ Readmission within 30 days after discharge } \\
\hline & No $(n=192)$ & Yes $(n=48)$ & $P$ value \\
\hline $\begin{array}{l}\text { Age, yrs, mean } \\
\text { (SD) }\end{array}$ & $63.0(13.6)$ & $59.9(14.5)$ & 0.163 \\
\hline $\begin{array}{l}\text { LOS, days, mean } \\
\text { (SD) }\end{array}$ & $11.2(11.6)$ & $9.7(7.6)$ & 0.420 \\
\hline Race & & & 0.743 \\
\hline White & $115(59.9)$ & $27(56.3)$ & \\
\hline Other & $77(40.1)$ & $21(43.8)$ & \\
\hline Gender & & & 0.020 \\
\hline Female & $60(31.3)$ & 7 (14.6) & \\
\hline Male & $132(68.8)$ & $41(85.4)$ & \\
\hline \multicolumn{4}{|l|}{ Comorbidity } \\
\hline $\mathrm{CAD}$ & $137(71.4)$ & $28(58.3)$ & 0.085 \\
\hline MI & $75(39.1)$ & $20(41.7)$ & 0.744 \\
\hline $\mathrm{DM}$ & $103(53.7)$ & $26(54.2)$ & 1.000 \\
\hline Hypertension & $156(81.3)$ & $38(79.2)$ & 0.838 \\
\hline COPD & $44(22.9)$ & $10(20.8)$ & 0.848 \\
\hline ICD & $100(52.1)$ & $22(45.8)$ & 0.519 \\
\hline LVAD & $8(4.2)$ & $2(4.2)$ & 1.000 \\
\hline $\begin{array}{l}\text { History of prior } \\
\text { stroke }\end{array}$ & $19(9.9)$ & $1(2.1)$ & 0.139 \\
\hline Obesity & $52(27.1)$ & $13(27.1)$ & 1.000 \\
\hline $\begin{array}{l}\text { At least one } \\
\text { comorbidity }\end{array}$ & $186(96.9)$ & $46(95.8)$ & 0.662 \\
\hline
\end{tabular}

Note. Numbers in the parenthesis are percentage except indicated.

level, or HGB between the two groups of patients in the univariate analysis (Table 2).

To further investigate the effect of each independent variable while controlling other covariates, multivariate analyses were performed (Table 3 and Figure 1). The results showed that the KCCQ score and EF were negatively associated with readmission rate (adjusted $\mathrm{OR}=0.566$ and 1.903 and $P=0.022$ and 0.021 , resp.) and that males were more likely to be readmitted than females (adjusted $\mathrm{OR}=5.589$, $P=0.001)$. Interestingly, patients with MI were more likely (adjusted $\mathrm{OR}=2.849, P=0.049$ ) and patients with CAD were less likely to be readmitted (adjusted OR $=0.231$, $P=0.012$ ), compared to patients with other comorbidities. One possible interpretation could be that patients who have had a myocardial infarction are more likely to have wall motion abnormalities and fixed myocardial defects and thus a lower ejection fraction than those with nonobstructive coronary artery disease without an MI, leading to opposite contribution to HF readmission.

In order to evaluate how much contribution the KCCQ score made in predicting HF readmission, we developed a model by including seven factors besides KCCQ score (model 5) based on the multivariate regression results, published literature, and models. The $c$-statistic indicated
TABLE 2: Summary of KCCQ score, lab tests, and discharge medication between HF readmission and nonreadmission within 30 days after discharge.

\begin{tabular}{|c|c|c|c|}
\hline \multirow{2}{*}{$\begin{array}{l}\text { Demographic } \\
\text { characteristics }\end{array}$} & \multicolumn{3}{|c|}{ Readmission within 30 days after discharge } \\
\hline & No $(n=192)$ & Yes $(n=48)$ & $P$ value \\
\hline $\begin{array}{l}\text { KCCQ score, } \\
\text { mean (SD) }\end{array}$ & $40.8(22.2)$ & $32.6(18.5)$ & 0.019 \\
\hline \multicolumn{4}{|l|}{$\begin{array}{l}\text { Lab on } \\
\text { admission }\end{array}$} \\
\hline $\begin{array}{l}\text { Sodium, mean } \\
\text { (SD) }\end{array}$ & $137.6(4.7)$ & $137.5(5.6)$ & 0.915 \\
\hline $\begin{array}{l}\text { HGB, mean } \\
(\mathrm{SD})\end{array}$ & $12.1(2.1)$ & $11.9(2.1)$ & 0.622 \\
\hline Ejection fraction & $24.7(7.4)$ & $21.8(8.8)$ & 0.021 \\
\hline \multicolumn{4}{|l|}{$\begin{array}{l}\text { Discharge } \\
\text { medication }\end{array}$} \\
\hline Beta blocker & $172(89.6)$ & $43(89.6)$ & 1.000 \\
\hline $\mathrm{ACE} / \mathrm{ARB}$ & $110(57.3)$ & $25(52.1)$ & 0.520 \\
\hline Diuretic & $168(87.5)$ & $40(83.3)$ & 0.478 \\
\hline Lipid-lowering & $126(65.6)$ & $29(60.4)$ & 0.504 \\
\hline $\begin{array}{l}\text { Aldosterone } \\
\text { antagonist }\end{array}$ & $98(51.0)$ & $23(47.9)$ & 0.748 \\
\hline Digoxin & $60(31.3)$ & $15(31.3)$ & 1.000 \\
\hline Hydralazine & $30(15.6)$ & $5(10.4)$ & 0.494 \\
\hline Nitrates & $39(20.3)$ & $6(12.5)$ & 0.301 \\
\hline Inotrope & $46(24.0)$ & $13(27.1)$ & 0.708 \\
\hline
\end{tabular}

Note. Numbers in the parenthesis are percentage except indicated.

that model 5 which included KCCQ score and all other potential predictors had the highest $c$-statistic value (0.710) among other reduced models without KCCQ score (Figure 2). As seen in Table 4, the IDI analysis demonstrated that the discriminatory performance of model 5 improved by $6.6 \%$ from the base model (model 1) that only included age and gender and by $2.7 \%$ from the reduced model (model 4 ) including all factors but the KCCQ score (this is the absolute increment; when compared with model 4, the IDI of the full model with KCCQ, model 5, increased by $2.7 / 3.9=69 \%$ ). On the other hand, as an established independent factor associated with HF readmission [12, 13], EF increased the IDI from $1.3 \%$ (model 3 ) to $3.9 \%$ (model 4 ). These results suggested that the KCCQ score, as a single independent variable, is one of the important factors that could potentially be used for predicting readmission rates of HF patients within 30 days after discharge, and a combination of all these important factors would offer the greatest incremental gain.

\section{Discussion}

In this prospective study, we found that the KCCQ score was significantly associated with short-term HF readmission rate. It contributed to improving the $c$-statistics of a model based on age, gender, medications, laboratory data, and LVEF available at discharge from 0.670 to 0.710 and raised the 
TABLE 3: Summary of multivariate analysis investigating the effects of demographic characteristics, medical history, discharge medication, lab test, and overall KCCQ score on readmission rate within 30 days after discharge $(n=240)$.

\begin{tabular}{|c|c|c|c|c|}
\hline Factor & Adjusted OR & SE & $95 \% \mathrm{CI}$ & $P$ value \\
\hline Age & 0.990 & 0.145 & $0.742-1.320$ & 0.946 \\
\hline White & 0.821 & 0.348 & $0.358-1.884$ & 0.642 \\
\hline Male & 5.589 & 2.962 & $1.979-15.79$ & 0.001 \\
\hline CAD & 0.231 & 0.135 & $0.074-0.724$ & 0.012 \\
\hline MI & 2.849 & 1.514 & $1.005-8.074$ & 0.049 \\
\hline $\mathrm{DM}$ & 0.877 & 0.369 & $0.384-2.001$ & 0.754 \\
\hline Hypertension & 0.815 & 0.405 & $0.308-2.157$ & 0.681 \\
\hline COPD & 1.084 & 0.514 & $0.429-2.744$ & 0.864 \\
\hline ICD & 0.648 & 0.271 & $0.286-1.471$ & 0.299 \\
\hline LVAD & 0.710 & 0.650 & $0.118-4.275$ & 0.709 \\
\hline History of prior stroke & 0.150 & 0.171 & $0.016-1.402$ & 0.096 \\
\hline Obesity & 1.377 & 0.658 & $0.540-3.511$ & 0.503 \\
\hline Beta blocker & 1.096 & 0.713 & $0.306-3.920$ & 0.888 \\
\hline $\mathrm{ACE} / \mathrm{ARB}$ & 0.734 & 0.299 & $0.331-1.629$ & 0.447 \\
\hline Diuretic & 0.438 & 0.257 & $0.138-1.384$ & 0.159 \\
\hline Lipid-lowering & 1.186 & 0.511 & $0.509-2.761$ & 0.693 \\
\hline Aldosterone antagonist & 0.873 & 0.360 & $0.389-1.957$ & 0.741 \\
\hline Digoxin & 1.137 & 0.47 & $0.506-2.554$ & 0.756 \\
\hline Hydralazine & 0.639 & 0.402 & $0.186-2.193$ & 0.476 \\
\hline Nitrates & 0.443 & 0.271 & $0.134-1.467$ & 0.182 \\
\hline Inotrope & 0.799 & 0.378 & $0.316-2.022$ & 0.636 \\
\hline Sodium & 1.791 & 0.815 & $0.734-4.368$ & 0.200 \\
\hline $\mathrm{Hgb}$ & 0.810 & 0.087 & $0.655-1.000$ & 0.050 \\
\hline Ejection fraction & 1.903 & 0.532 & $1.100-3.292$ & 0.021 \\
\hline KCCQ & 0.566 & 0.141 & $0.347-0.922$ & 0.022 \\
\hline
\end{tabular}

TABle 4: Prognostic value of readmission within 30 days after discharge of different models comparing to model 1 with only demographic predictors.

\begin{tabular}{|c|c|c|c|}
\hline Model & $c$-statistics & IDI increase (\%) & $P$ value \\
\hline Model 1: age + gender & 0.617 & - & - \\
\hline Model 2: age + gender + beta_blocker + ace/arb & 0.647 & 0.9 & 0.123 \\
\hline Model 3: age + gender + beta_blocker + ace/arb + sodium + hgb & 0.656 & 1.3 & 0.081 \\
\hline Model 4: age + gender + beta_blocker + ace/arb + sodium + hgb + ef & 0.670 & 3.9 & 0.005 \\
\hline Model 5: age + gender + beta_blocker + ace/arb + sodium + hgb + ef + KCCQ & 0.710 & 6.6 & $<0.001$ \\
\hline
\end{tabular}

IDI by $2.7 \%$, which suggested that it may be helpful in predicting 30-day readmission and thus significantly improve prediction reliability when combined with other critical components. These findings may provide some help to guide follow-up strategies towards delivering optimal care, such as encouraging patients with lower KCCQ to have an early follow-up [14].

Lots of efforts have been made to identify the predictable factors that are associated with high risk of being readmitted, which has been quite challenging until now. In this study, we found that HF patients who had lower KCCQ score at time of discharge and lower EF and of male gender seemed to be more prone for readmission within 30 days. These findings were similar to some studies but not others. As a matter of fact, no specific patient or hospital factors have been shown to consistently predict 30-day readmission after hospitalization for HF. In a systematic review of 112 studies describing the association between traditional patient characteristics and readmission after hospitalization for $\mathrm{HF}$, left ventricular $\mathrm{EF}$, as well as other factors such as demographic characteristics, comorbid conditions, and New York Heart Association class, was associated with readmission in only a minority of cases [13]. In another meta-analysis of 69 studies and 144 factors for short-term readmission, noncardiovascular comorbidities, poor physical condition, history of admission, and failure to use evidence-based medication, rather than cardiovascular comorbidities, age, or gender, were more strongly associated with short-term readmission [15].

The KCCQ scores have been demonstrated to have much greater sensitivity to clinical changes in HF patients than 


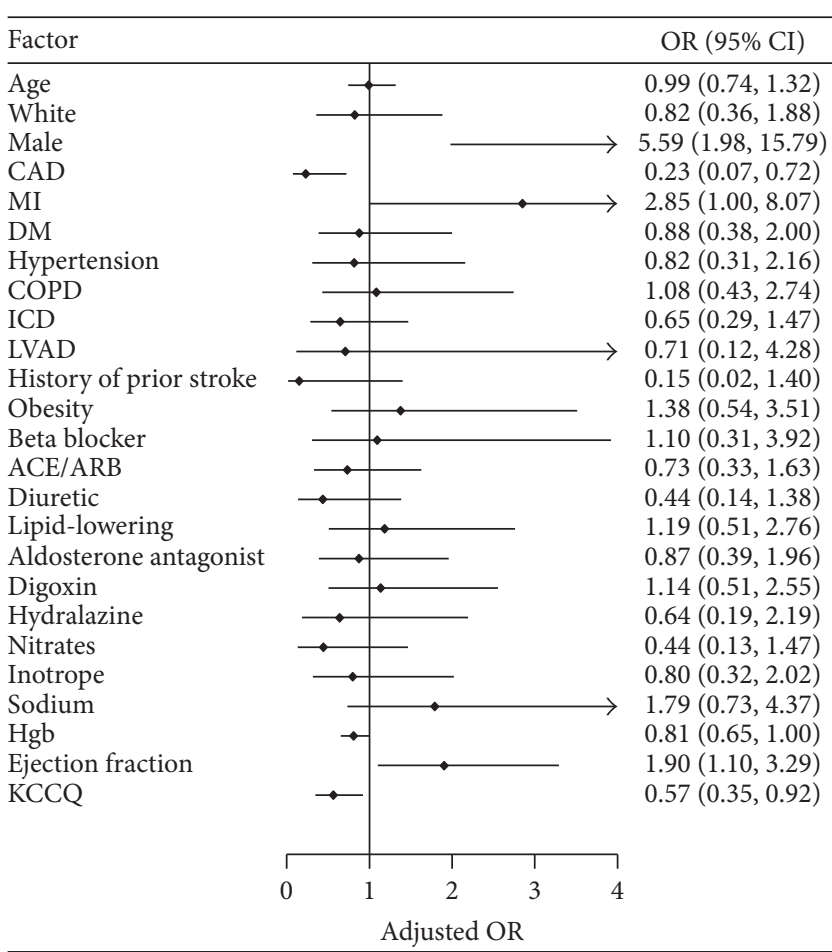

FIGURE 1: Adjusted odds ratios of readmission within 30 days after discharge derived from multivariate logistic regression analysis.

the New York Heart Association (NYHA) functional classification, Minnesota Living with Heart Failure Questionnaire (LiHFe), and Short Form-36 (SF-36) [6]. The EVEREST trial suggested that the KCCQ is an important prognostic indicator of readmission within one year after discharge [9]. In their study, patients with KCCQ scores $<25$ (worse health status) had more than threefold increased risk of the combined endpoint of rehospitalization and mortality than those in the best health status tier (KCCQ score > 75). More recently, KCCQ score was used to assess the feasibility of reflecting the changes of acute HF during hospitalization and predicting 30-day readmission. The authors found that it was feasible to use the KCCQ during acute HF hospitalizations and was sensitive to clinical improvement, but score changes during hospitalization did not predict 30 -day readmission. However, this study was a relatively small study that included only 54 patients and was focused on KCCQ score differences during hospitalization between nonreadmission and admission groups [10]. In contrast, more than 240 patients were enrolled in our study and the KCCQ score was higher in nonreadmitted HF patients and was independently associated with lower 30-day readmission.

As mentioned above, there are multiple factors contributing to HF readmission; therefore, risk prediction models including and weighing all relevant factors were developed. In these models, discrimination, defined by the area under the receiver operating characteristic (ROC) curve, is used to tell how well a model can separate those who will have the outcome from those who will not have the outcome of interest. In this case, if the predicted risks

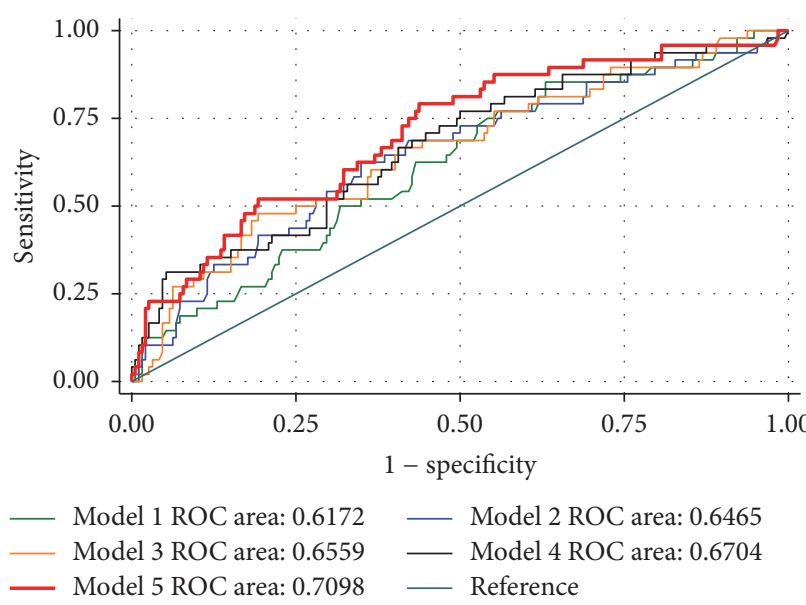

FIgure 2: Comparison of ROC area among different models. Model 1: $\operatorname{logit}(\operatorname{read} 30)=$ age + gender; $\operatorname{model} 2: \operatorname{logit}(\operatorname{read} 30)=$ age + gender + beta_blocker + ace/arb; model 3: logit $(\operatorname{read} 30)=$ age + gender + beta_blocker + ace/arb + sodium + hgb; model 4: logit $(\operatorname{read} 30)=$ age + gender + beta_blocker + ace/arb + sodium + hgb + ef; and model 5: logit $($ read30 $)=$ age + gender + beta_blocker + ace/arb + sodium + hgb + ef + KCCQ. read30: readmission in 30 days.

for readmitted patients are all higher than for patients who are not readmitted, the model discriminates perfectly with $c$-statistic of 1 . Conversely, if risk prediction is no better than chance, the $c$-statistic is 0.5 . Models are typically considered reasonable when the $c$-statistic is greater than 0.7 and strong when the $c$-statistic is greater than 0.8 [16]. For 30-day readmission after HF hospitalization, several models have been developed. Only two models have generated $c$-statistics greater than 0.6 after studying both derivation and validation cohorts. One of them is the automated model developed by Amarasingham et al. incorporating data from the electronic health record at the time of hospitalization [17]. The other model combined claims-based demographic and comorbidity data with clinical data including vital signs, laboratory values, and measured left ventricular ejection fraction [18]. However, neither of the two models included KCCQ scores. Given only 48 readmissions in our study population, we included only 7 parameters besides the KCCQ score in the full model (model 5). Low EF and gender (men) resulting in increased odds ratios for readmission in the multivariate analysis were included; we also included information about medications, beta-blocker and ACE inhibitor/ARB, which had demonstrated lowering HF mortality [19-21], and sodium and $\mathrm{Hgb}$, which may affect $\mathrm{HF}$ rehospitalization and mortality $[22,23]$ and have been used in other models (http://www.readmissionscore.org/heart_failure.php), although they were not independently associated with readmission in the multivariate analysis. The full model (model 5), which included the KCCQ score, increased the $c$-statistics of 0.617 in base model 1 based on age and gender to 0.710 , with an IDI increase of $6.6 \%$. Given that many other possible risk factors have not been included in 
this model, such as GFR and BNP, this model may not be perfect, although its $c$-statistics was greater than 0.7 , and may exaggerate the contribution of the KCCQ score. However, our results suggested that the contribution of KCCQ for predicting short-term HF readmission could potentially be as important as LVEF.

The present findings should be considered within the context of the study's limitations. This study was performed in a single-community medical center, and further studies in other centers or multiple centers need to be done to validate our findings. We only administered the KCCQ one time during the hospitalization, which would not reflect changes between admission, during hospitalization, and after hospitalization. We did not collect some relevant medical history, such as history of admission due to heart failure in the past; physical examination findings; some other labs such as GFR and BNP, or chest X-ray findings. These factors could also be important in the risk prediction model.

\author{
Abbreviations \\ KCCQ: Kansas City Cardiomyopathy Questionnaire \\ HF: Heart failure \\ HRQL: Health-related quality of life \\ EF: $\quad$ Ejection fraction \\ LVEF: Left ventricular ejection fraction \\ OR: Odds ratios \\ CAD: Coronary artery disease \\ MI: Myocardial infarction \\ DM: Diabetes mellitus \\ COPD: Chronic obstructive pulmonary disease \\ ICD: Implantable cardioverter-defibrillator \\ LVAD: Left ventricular assist device \\ ACE: Angiotensin converting enzyme \\ ARBs: Angiotensin receptor blockers \\ HGB: Hemoglobin \\ IDI: Integrated discrimination improvement \\ NYHA: New York Heart Association \\ ROC: Receiver operating characteristic \\ GFR: Glomerular filtration rate \\ BNP: Brain natriuretic peptide.
}

\section{Additional Points}

Competency in Medical Knowledge. Heart failure is one of the most common diagnoses associated with readmission. KCCQ score provided important prognostic information for predicting 30-day readmission and it can significantly improve prediction reliability along with other critical components. Translational Outlook. Additional clinical studies need to be done in multiple centers with a larger sample size to validate our finding. Future research should include relevant physical examination findings and chest X-ray findings, which could be important in the risk prediction model.

\section{Competing Interests}

The authors declare that they have no competing interests.

\section{Authors' Contributions}

Shengchuan Dai and Junhong Gui contributed equally to the manuscript.

\section{Acknowledgments}

The authors wish to acknowledge the following participating doctors from Florida Hospital Orlando who helped with data collection: Maria Amin (MD); Saroj Khadka (MD); Prabhava Bagla (MD); and Zoltan Varga (MD, Ph.D.).

\section{References}

[1] D. Mozaffarian, E. J. Benjamin, A. S. Go et al., "Heart disease and stroke statistics-2015 update: a report from the american heart association," Circulation, vol. 131, no. 4, pp. e29-e322, 2015.

[2] S. F. Jencks, M. V. Williams, and E. A. Coleman, "Rehospitalizations among patients in the medicare fee-for-service program," The New England Journal of Medicine, vol. 360, no. 14, pp. 14181428, 2009.

[3] H. M. Krumholz, J. Amatruda, G. L. Smith et al., "Randomized trial of an education and support intervention to prevent readmission of patients with heart failure," Journal of the American College of Cardiology, vol. 39, no. 1, pp. 83-89, 2002.

[4] G. Gerhardt, A. Yemane, P. Hickman, A. Oelschlaeger, E. Rollins, and N. Brennan, "Medicare readmission rates showed meaningful decline in 2012," Medicare and Medicaid Research Review, vol. 3, no. 2, 2013.

[5] K. Dharmarajan, A. F. Hsieh, Z. Lin et al., "Diagnoses and timing of 30-day readmissions after hospitalization for heart failure, acute myocardial infarction, or pneumonia," The Journal of the American Medical Association, vol. 309, no. 4, pp. 355-363, 2013.

[6] C. P. Green, C. B. Porter, D. R. Bresnahan, and J. A. Spertus, "Development and evaluation of the Kansas City Cardiomyopathy Questionnaire: a new health status measure for heart failure," Journal of the American College of Cardiology, vol. 35, no. 5, pp. 1245-1255, 2000.

[7] G. E. Soto, P. Jones, W. S. Weintraub, H. M. Krumholz, and J. A. Spertus, "Prognostic value of health status in patients with heart failure after acute myocardial infarction," Circulation, vol. 110, no. 5, pp. 546-551, 2004.

[8] P. A. Heidenreich, J. A. Spertus, P. G. Jones et al., "Health status identifies heart failure outpatients at risk for hospitalization or death," Journal of the American College of Cardiology, vol. 47, no. 4, pp. 752-756, 2006.

[9] S. M. Dunlay, M. Gheorghiade, K. J. Reid et al., "Critical elements of clinical follow-up after hospital discharge for heart failure: insights from the EVEREST trial," European Journal of Heart Failure, vol. 12, no. 4, pp. 367-374, 2010.

[10] K. Sauser, J. A. Spertus, L. Pierchala, E. Davis, and P. S. Pang, "Quality of life assessment for acute heart failure patients from emergency department presentation through 30 days after discharge: a pilot study with the kansas city cardiomyopathy questionnaire," Journal of Cardiac Failure, vol. 20, no. 1, pp. 1822, 2014.

[11] M. J. Pencina, R. B. D’Agostino, and R. B. D’Agostino Jr., "Evaluating the added predictive ability of a new marker: from area under the roc curve to reclassification and beyond," Statistics in Medicine, vol. 27, no. 2, pp. 157-172, 2008. 
[12] K. J. Harjai, E. Nunez, T. Turgut et al., “The independent effects of left ventricular ejection fraction on short-term outcomes and resource utilization following hospitalization for heart failure," Clinical Cardiology, vol. 22, no. 3, pp. 184-190, 1999.

[13] J. S. Ross, G. K. Mulvey, B. Stauffer et al., "Statistical models and patient predictors of readmission for heart failure: a systematic review," Archives of Internal Medicine, vol. 168, no. 13, pp. 13711386, 2008.

[14] K. K. Lee, J. Yang, A. F. Hernandez, A. E. Steimle, and A. S. Go, "Post-discharge follow-up characteristics associated with 30-day readmission after heart failure hospitalization," Medical Care, vol. 54, no. 4, pp. 365-372, 2016.

[15] M. Saito, K. Negishi, and T. H. Marwick, "Meta-analysis of risks for short-term readmission in patients with heart failure," The American Journal of Cardiology, vol. 117, no. 4, pp. 626-632, 2016.

[16] D. W. Hosmer Jr., S. Lemeshow, and R. X. Sturdivant, Eds., Applied Logistic Regression, John Wiley \& Sons, New York, NY, USA, 2nd edition, 2000.

[17] R. Amarasingham, B. J. Moore, Y. P. Tabak et al., "An automated model to identify heart failure patients at risk for 30-day readmission or death using electronic medical record data," Medical Care, vol. 48, no. 11, pp. 981-988, 2010.

[18] B. G. Hammill, L. H. Curtis, G. C. Fonarow et al., "Incremental value of clinical data beyond claims data in predicting 30day outcomes after heart failure hospitalization," Circulation: Cardiovascular Quality and Outcomes, vol. 4, no. 1, pp. 60-67, 2011.

[19] J. M. Foody, M. H. Farrell, and H. M. Krumholz, “ $\beta$-Blocker therapy in heart failure: scientific review," The Journal of the American Medical Association, vol. 287, no. 7, pp. 883-889, 2002.

[20] M. D. Flather, S. Yusuf, L. Kober et al., "Long-term ace-inhibitor therapy in patients with heart failure or left-ventricular dysfunction: a systematic overview of data from individual patients. Ace-inhibitor myocardial infarction collaborative group," The Lancet, vol. 355, no. 9215, pp. 1575-1581, 2000.

[21] M. A. Pfeffer, J. J. V. McMurray, E. J. Velazquez et al., "Valsartan, captopril, or both in myocardial infarction complicated by heart failure, left ventricular dysfunction, or both," The New England Journal of Medicine, vol. 349, no. 20, pp. 1893-1906, 2003.

[22] A. Romanovsky, S. Bagshaw, and M. H. Rosner, "Hyponatremia and congestive heart failure: a marker of increased mortality and a target for therapy," International Journal of Nephrology, vol. 2011, Article ID 732746, 7 pages, 2011.

[23] Y.-D. Tang and S. D. Katz, "Anemia in chronic heart failure: prevalence, etiology, clinical correlates, and treatment options," Circulation, vol. 113, no. 20, pp. 2454-2461, 2006. 


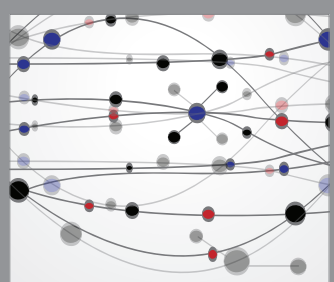

The Scientific World Journal
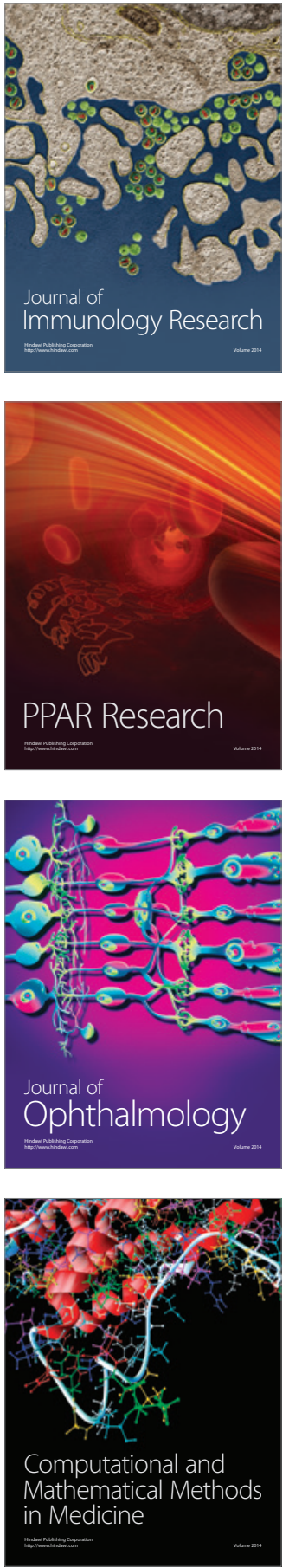

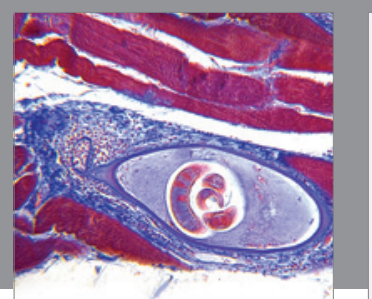

Gastroenterology Research and Practice

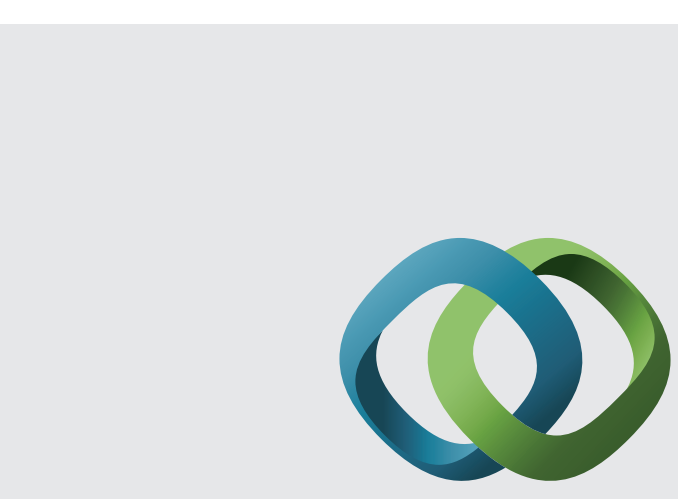

\section{Hindawi}

Submit your manuscripts at

http://www.hindawi.com
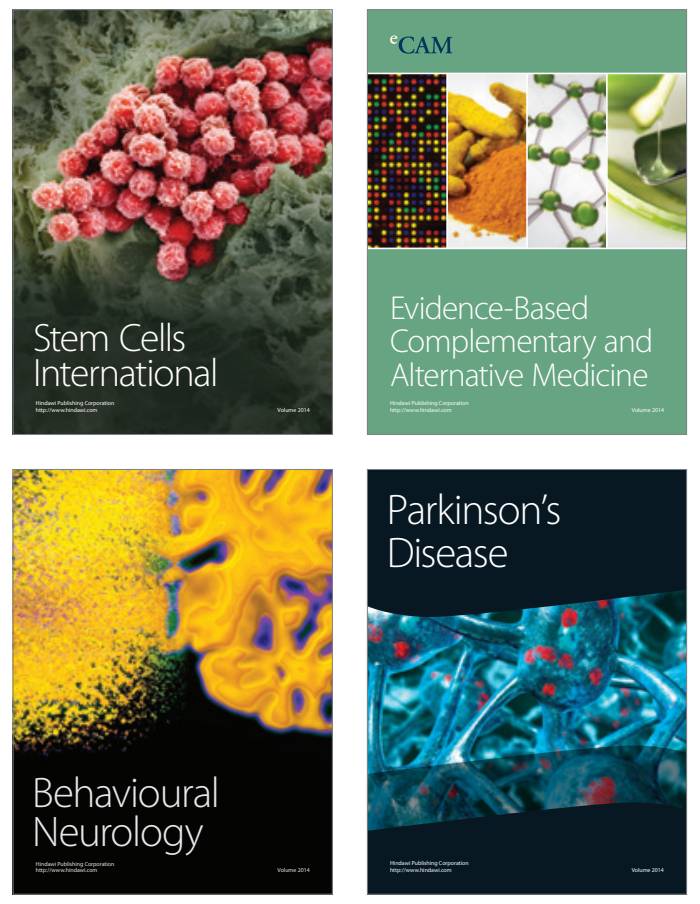
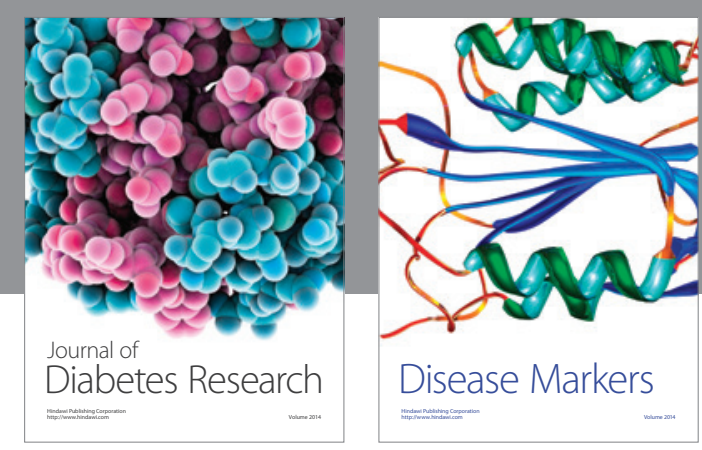

Disease Markers
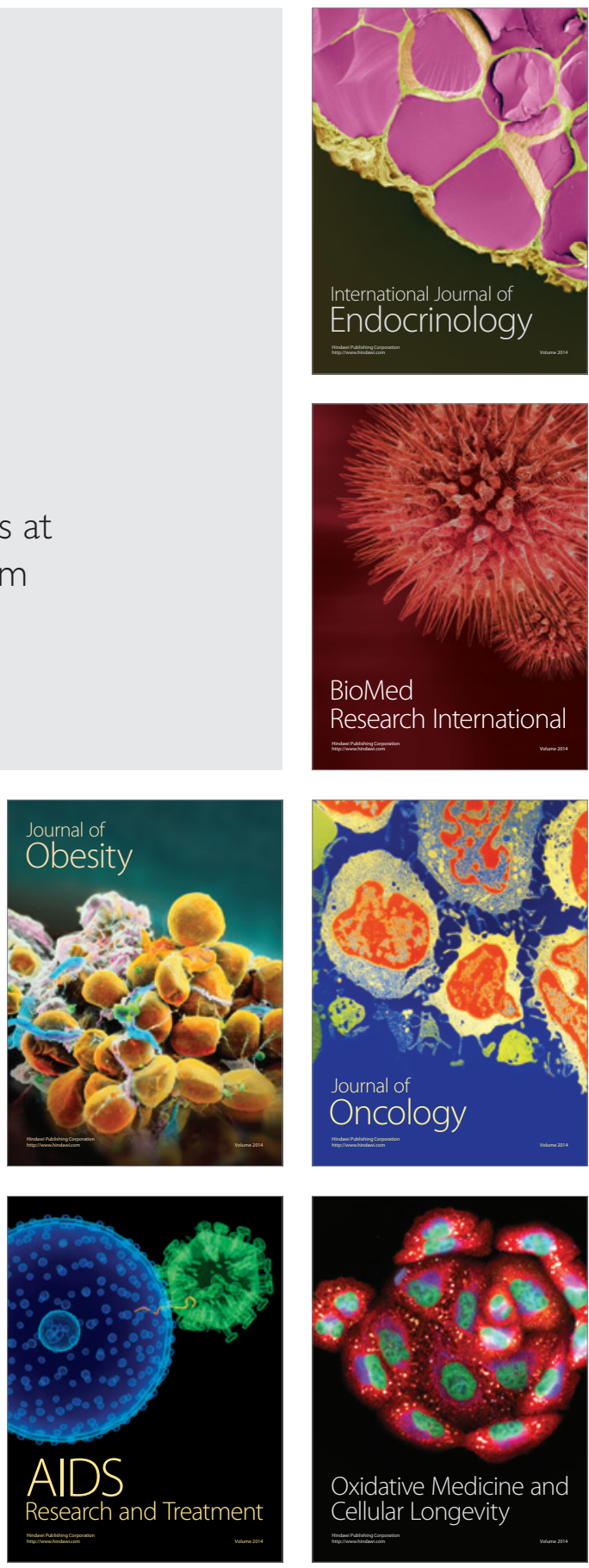